\title{
Anti-CD40/Anti-TAA Bispecific Monoclonal Antibody ABBV-428
}

National Cancer Institute

\section{Source}

National Cancer Institute. Anti-CD40/Anti-TAA Bispecific Monoclonal Antibody ABBV-

428. NCl Thesaurus. Code C132681.

\begin{abstract}
A bispecific monoclonal antibody composed of a binding domain for an epitope found on the cell-surface receptor CD40 linked to a binding domain directed to an as of yet undisclosed tumor-associated antigen (TAA), with potential immunomodulating and antineoplastic activities. Upon administration of anti-CD40/anti-TAA bispecific monoclonal antibody ABBV-428, the anti-TAA moiety targets and binds to the TAA expressed on the tumor cells. The agonistic anti-CD40 moiety targets and binds to various CD40-expressing immune cells. This leads to the activation and proliferation of effector and memory T-cells, and enhances the immune response against tumor cells, which kills and inhibits the proliferation of the TAA-expressing tumor cells. CD40, a stimulatory receptor and a member of the tumor necrosis factor receptor superfamily (TNFRSF), is expressed on various immune cells, such as macrophages, B-lymphocytes, and dendritic cells (DCs); it plays a key role in the activation of the immune system.
\end{abstract}

